\title{
The impact of the attachment implementation in the healthcare domain
}

\author{
Hicham Amine ${ }^{1}$, Abdelouahab Mesnaoui ${ }^{2}$,Safaa Elkhaddam ${ }^{3}$ \\ 1 Department of Operations Management, Al Akhawayn University, Ifrane, Morocco \\ 2 E-Learning Consulting Groups, Rabat, Morocco \\ 3University of ibn tofail, faculty of science, laboratory of genetics and biometry
}

\begin{abstract}
An "attachment" is any supplemental health documentation needed from a provider to support a specific business process in the Healthcare domain while using electronic exchange within the organization or between healthcare companies and payer and/or providers. The purpose of this study is to enhance the submission of the healthcare transactions by adding an attachment for each transaction. This study includes the submission of medical documentation to support; that includes Health care claims, Appeals, Preauthorizations for services, Referral authorizations, Enrollee eligibility inquiries and Coordination of benefits. An attachment can be 'solicited' via a request from the provider or 'unsolicited' (sent pro-actively by the provider and not tied to a health plan request.) The majority of Attachments are solicited vs. unsolicited. We can notice a high difference between the ones are for and the ones against by comparing $70 \%$ and $30 \%$ which clarifies how this implementation will make the companies achieve a better and effective exchange of info which can improve the quality of the services.

Our study showed also that between 5-20\% of claims submissions require attachment information and that 30 to $45 \%$ of healthcare companies were able to feel an improvement in the transactions between the payer and the provider. And also 45-55\% of users welcomed the upgrade of the new change by adding an attachment to the transactions.

Implementation of the attachment as a new functionality was able to facilitate the exchange of information and effective communication between parties.
\end{abstract}

Key words: Attachment-healthcare-information

\section{Introduction}

A health care claims attachment conveys supplemental information pertaining to the services provided to a specific individual to support evaluation of a claim before it is paid [1]. An attachment might contain biometric data; medical history; clinical data (reports, studies, notes); hospital discharge notes; laboratory results; medication information; rehabilitation plans; optical prescriptions; certifications made by the individual and/or the health care provider regarding sterilization, hysterectomy, or other services, as required by Federal or State rules; or other clarifying information for a particular service [2]. Attachments may be requested or submitted when the supplemental medical information is directly related to the determination of benefits under the subscriber's contract, or when directly related to providing medical justification for health care services provided to the individual when that medical justification can affect the adjudication of payment for services billed by the provider of health care services. Although additional clinical or administrative information may be required following adjudication of claims, such as for post-adjudication review to support quality control, fraud and abuse, or other post-adjudication reviews and reporting requirements, we do not consider these postadjudication requests for claims-related data to be part of the claims payment process. Entities may voluntarily choose to use the standard transaction format and structure for requesting and submitting these types of attachments, those transactions are not considered electronic claims attachments [2]. The purpose of this study is to identify the impact an attachment might have on the exchange of requests and responses between the payer and the provider in the healthcare domain keeping in mind the HIPAA compliances that both payer and provider are required to follow besides other constraints they might face.

\section{Methodology}

A study was conducted regarding the implementation of attachment functionality when submission of medical documentation to support Health care claims, Appeals, Pre-authorizations for services, Referral authorizations, Enrollee eligibility inquiries and Coordination of benefits by distributing surveys to users from healthcare companies with no attachment option deployed.

The proposed answers were divided into two categories: 
- Related to operations department responsible of submitting requests to third party vendors or payers where we notified the positive attitude toward the proposed option of electronic attachment since operations will be much easier and accurate between entities.

- Related to IT department who is in charge of transmitting the responses received from the provider and we noticed a high motivation from employees to move from manual to automate system because it will lead to time and costs saving.

An attachment can be 'solicited' via a request from the provider or 'unsolicited' (sent pro-actively by the provider and not tied to a health plan request.) The majority of Attachments are solicited vs. unsolicited.

\section{Problem description}

\section{Results}

\section{Methods in use today}

In today's environment, most attachment exchanges (requests and submissions) are conducted sending hardcopies via U.S. mail, fax, and phone; a few are done electronically through secure portals offered by plans (to upload documentation) or via electronic transactions. This is due to the fact that not all the information required to be submitted to a health plan in an attachment is available electronically today. Only $30-40 \%$ of healthcare data reside in EHRs. Providers may only have access to 'paper' or 'imaged' reporting since not all of the data that exist in electronic form are maintained in coded, structured, and computable form. If codified data is available, exchange with a health plan may not be reliable as there are differences in code sets and structures between technologies and trading partners. The following scenarios depict the most common processes for requests and responses for additional claim information:

The below diagram shows the process that a provider claim submission which is pended at the health plan while the request is sent to the provider for additional information. The claim is in a pended state for up to 60 days while the provider sends back the information to be matched to the original claim.

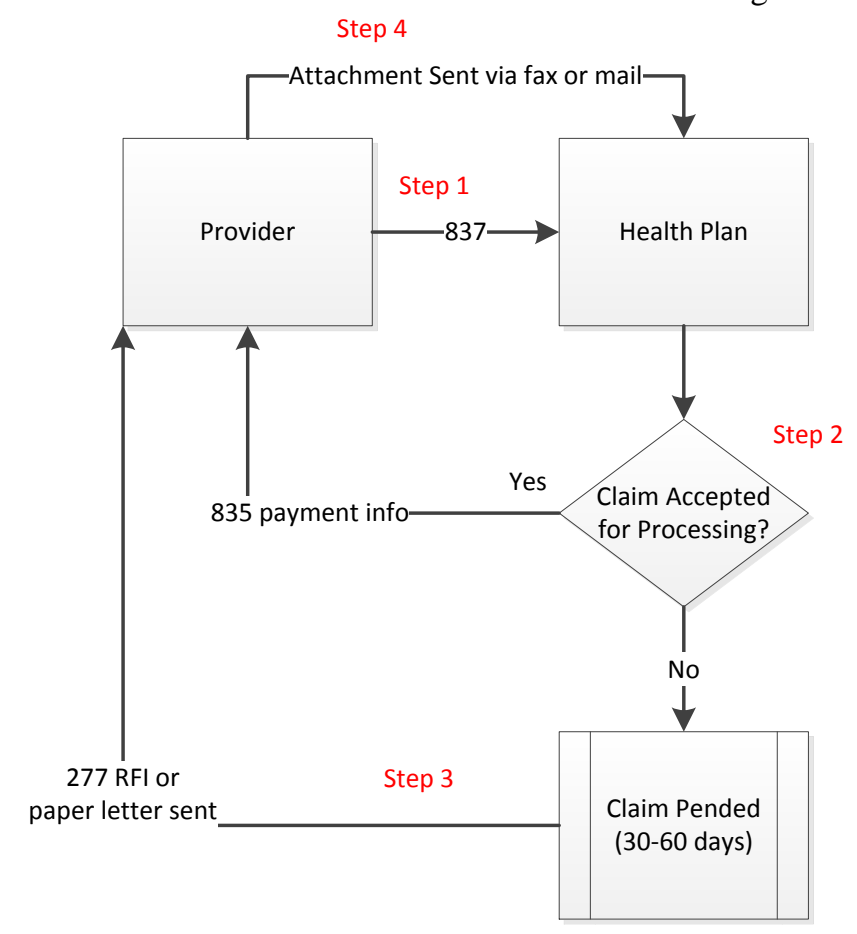

Fig.1. Process of Provider Submission with a pending claim

The health plan denies the submission and communicates the information required for re-submission via the 835 (CARC and RARC codes). Processing of the original claim and the subsequent re-submission can extend the time to payment for an additional $60-90$ days.

The below diagram shows the provider submission with a denial claim. 


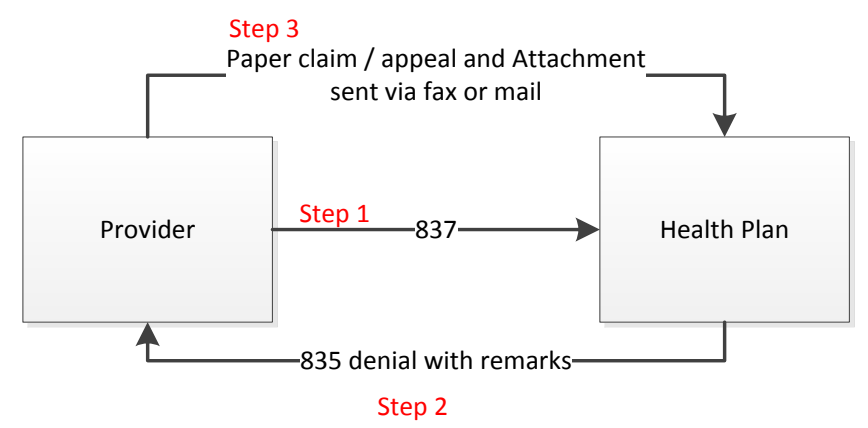

Fig2. Process of the provider submission with a denial claim

The proposed Healthcare Attachment Solution is designed to streamline the workflow of exchanging clinical and administrative information between providers and payers. The solution will provide end-to-end transaction visibility for payers to better manage supplemental healthcare information from providers. The attachment solution supports payer's ability in the exchange of electronic requests and responses with trading partner providers. Payers can accelerate the business processes associated with managing supplemental information for claims, authorizations, and other iterative activities they engage in with providers resulting in (30-40\% reduction in costs related to sorting, storage, administration and tracking)

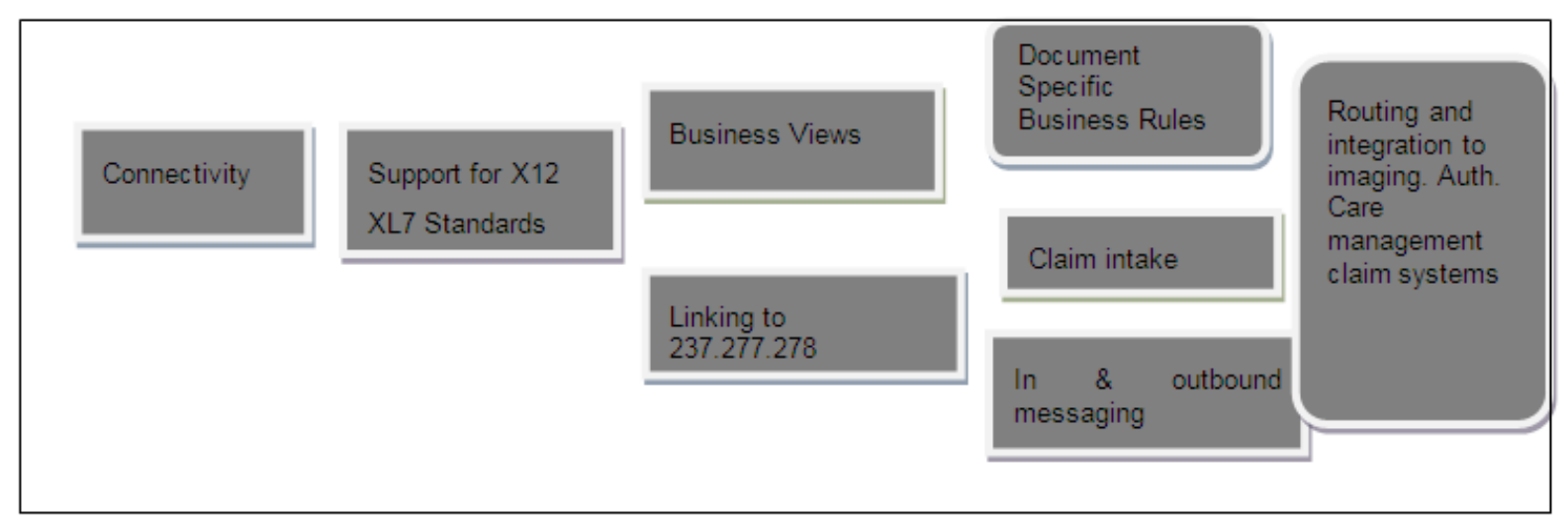

\section{Processing:}

Fig.3: Processing flow of inbound and outboud claims.

\section{Identification}

The Originator, Sender, Receiver, and Destination Partners of the incoming attachment, individual claims and prior authorizations are identified based on the inbound Trade Relationship.

\section{Validation}

The system validated incoming X12, XML, and HL7 based transactions.

\section{Loading to transaction manager}

The system can receive 837-claim, 275-attachments, 278-prior authorization and other attachments (with required metadata) files from a variety of sources and load it to transaction manager. The system would support the ability of the payer to process both the variants of exchanging attachment information i.e. human readable as well as codified data in the in the HL7 CDA that is carried in the BIN segment of the X12 275. The 6 attachment types supported are emergency department, rehabilitation services, laboratory results, ambulance medications, and clinical reports.

\section{Outbound Requests Acknowledgements}

Allow the payer's internal system to determine the need for an Attachment and provide a tool to create to outbound request.

\section{Linking:}

The solution supports payer's ability to electronically request additional information via 277RFI in the case of claims $(837 \mathrm{X12})$ or 278-Response in case 278-Request. The system tracks the outbound 277RFI and 278Response and once the attachments are received in response to the request for additional information the system links them to existing related transactions. 


\section{Discussion}

Attachment Solution is designed to streamline the workflow of exchanging clinical and administrative information between providers and payers. The solution will provide end-to-end transaction visibility for payers to better manage supplemental healthcare information from providers. The attachment solution supports payer's ability in the exchange of electronic requests and responses with trading partner providers. Payers can accelerate the business processes associated with managing supplemental information for claims, authorizations, and other iterative activities they engage in with providers resulting in (30-40\% reduction in costs related to sorting, storage, administration and tracking).

The solution provides Payers the ability to accept the inbound attachments via - EDI gateway, web portal, fax server or "e-mailbox". The system also maps the inbound X12 transaction and other compatible formats to the XML based format and stores it in the Claim Repository. The size of attachments can vary from $1 \mathrm{MB}$ to $190 \mathrm{MB}$. We could expect that the attachments would be received in context of multiple business transaction.

This study is showing how attachment option implementation can improve the transmission and exchange of info from either the submitter or the requestor specifically and the healthcare system in general. Another study conducted in UK came to that Modern healthcare systems are the epitome of complexity $[3,4,5]$. This presents challenges to researchers who evaluate and synthesize interventions attempting to improve healthcare systems and to policy makers who implement their findings [6].

We question whether the labors involved in reviewing particular healthcare interventions inform us sufficiently about the business of improving healthcare systems [6].

Our study also showed how attachment implementation will improve customer satisfaction because electronic submissions and/or requests will be free of errors and will lead to a modern healthcare system and will improve the e- healthcare system. Same result was found in a study done in Taiwan where it states that:" eHealthcare has increasingly taken shape in various forms in our surroundings and gained academic attention of researchers from different disciplines, including the medical, public health, technological, and innovation management domains. This reflects the fact that e-healthcare by nature entails cross-fertilization of medical informatics, public health and business [7]. From the medical field, much attention has been paid to the way an defectiveness of e-healthcare to improve the quality and well being of the service recipient, patients in particular $[8,9]$.

The Do IT as an R\&D funding agency with mandates of industrial innovation and development has sponsored e-healthcare projects led by the private sector, with an aim to promote Taiwan as a test- bed for innovative services. To this aim, the Do IT has committed to the view that its sponsorship to e-healthcare is meant to promote service innovations eventually leading to self-sustained business [10]. E-healthcare has a strong "outside-in" flavor, with new technologies and platforms medical experts to outreach and take care of the customer/cared living and behaving outside the medical premises in a smarter and proactive way [11]. This study also provided feedback from users who were concerned about security and they stated that sending and/or receiving attachments electronically will increase the security and privacy of the data of the patients that has to be confidential. Same result was found in a research done in Italy where they showed that: "we apply such facilities on MTOM attachments, in order to ensure also the security of large-sized imaging data. We remark that before data transfer, it is carried out the certificate-based mutual authentication between endpoints"[12].

The attachment implementation will lead to a modern healthcare system in terms of exchange of info in the claim submission either for an accepted or a denied claim to better judge the findings in the payer and/or the provider side. To improve healthcare systems and to policy makers who implement their findings. We question whether the labors involved in reviewing particular healthcare interventions inform us sufficiently about the business of improving healthcare systems [13].

This implementation has to be deployed at the earliest convenience since the process takes too long to be done with free bugs. In a study done in the UK, it was stated that" We also observe that in the longue dure of implementation these interventions become more sensitive to the need for whole system change and thus revise our model accordingly [14].

Healthcare systems have to be improved which attachment is part of which will take the healthcare system to the next level. Many questions and concerns were part of a study performed in the UK where it's stated that "what happens when there is a formal intervention seeking system improvement? Can we expect navigation and control of the new ideas to be maintained across the system layers? This latter question is the supreme challenge for all investigations of service improvement programs [15].

\section{Conclusion}

Attachment implementation is an important functionality that has to be added as soon as possible due to the high volume of transactions between the payers and providers. This option will allow a quick, efficient exchange of information in a safe and secure platform which saves the patient health info from any fraud. 
Through our study, we were able to show the high significant impact on the healthcare domain based on the results above that encourage the move from manual to an automate system taking the industry to the next level of performance. Based on the results got above, we are urging all healthcare organizations to move forward and initiate the need of an electronic attachment with an effective action plan ready for deployment.

\section{References bibliographiques}

[1]. Pawson, R.; The Science of Evaluation: a Realist Manifesto. Sage, London, (2013).

[2]. Federal register; the daily journal of the united state government; health and human department ; (2005).

[3]. Best A., Greenhalgh T., Lewis S., Sau J.E., Carroll, S., Bitz, J.; Large-system transformation in health care: a realist review. Millbank Q. 90, 421- 456, (2012).

[4]. Clark, A.; What are the component of complex interventions? Theorizing approaches to parts, powers and the whole intervention. Soc. Sci. Med. 93, 185-193, (2013).

[5]. Thomson S., Osborn R., Squires D., Jun M., International Profiles of Health Care Systems, The Commonwealth Fund, New York, (2012).

[6]. Ray P. , Joanne G., Cathy B. , Elizabeth G.; Do reviews of healthcare interventions teach us how to improvehealthcare systems. Social Science \& Medicine 114,129-137, (2014).

[7]. Eysenbach G.; What is e-health? J. Med. Internet Res. 3 (2), 20, (2001).

[8]. Hood L.,Friend S.H.; Predictive,personalized, preventive,participatory(P4), cancer medicine.Nat.Rev.Clin.Oncol.8(3),184-187,( 2011).

[9]. Free C., Phillips G., Watson L., Galli L., Felix L., Edwards P., Patel V., Haines A.,. The effectiveness of mobile-health technologies to improve health care service delivery processes: a systematic review and meta-analysis. (2013).

[10]. Shin-Horng C., Pei-ChangWen N., Chih-Kai Y.; Business concepts of systemic service innovations in e-Healthcare, in press

[11]. Arcangelo C. , Raffaele P. , De Santis A., Carpentieri B. , Castiglione A., Palmieri F.; Cloud-based adaptive compression and secure management services for 3D healthcare data, (2013).

[12]. Clark, A.;What are the component of complex interventions? Theorizing?approaches to parts, powers and the whole intervention. Soc. Sci. Med. 93,185e193 (2013).

[13]. Hendry, C.; Understanding and creating whole organizational change through learning theory. Hum. Relations 49, 621e641, (1996).

[14]. Thomson, S., Osborn, R., Squires, D., Jun, M., International Profiles of Health Care Systems, The Commonwealth Fund, New York. (2012).

[15]. Eaton, L., BMA backs GPs in their objections to financial incentives to limit hospital referrals. Br. Med. J. 337, a2306, (2008). 